\title{
Amygdalin ameliorates the progression of atherosclerosis in LDL receptor-deficient mice
}

\author{
JIANZHEN LV ${ }^{1 *}$, WEN XIONG ${ }^{2 *}$, TIANTIAN LEI $^{3 *}$, HAILIAN WANG $^{4}$, MINGHAN SUN $^{5}$, ERWEI HAO ${ }^{1}$, \\ ZHIPING WANG ${ }^{1}$, XIAOQI HUANG ${ }^{6}$, SHAOPING DENG $^{4}$, JIAGANG DENG $^{1}$ and YI WANG ${ }^{7}$
}

${ }^{1}$ Guangxi Key Laboratory of Efficacy Study on Chinese Materia Medica, Guangxi University of Chinese Medicine, Nanning, Guangxi 530200; ${ }^{2}$ Department of Ultrasound, Sichuan Academy of Medical Science and Sichuan Provincial People's Hospital, Chengdu, Sichuan 610072; ${ }^{3}$ Department of Pharmacy, Medical School of University of Electronic Science and Technology of China, Chengdu, Sichuan 610054; ${ }^{4}$ Institute of Organ Transplantation; ${ }^{5}$ Department of Gynecology, Sichuan Academy of Medical Science and Sichuan Provincial People's Hospital, Chengdu, Sichuan 610072; ${ }^{6}$ Affiliated Hospital of Guangxi Traditional Chinese Medical University, Nanning, Guangxi 530001; ${ }^{7}$ Department of Pharmacy, Sichuan Academy of Medical Science and Sichuan Provincial People's Hospital, Chengdu, Sichuan 610072, P.R. China

Received January 23, 2017; Accepted August 15, 2017

DOI: $10.3892 / \mathrm{mmr} .2017 .7609$

\begin{abstract}
Previous studies have demonstrated that regulatory $\mathrm{T}$ cells (Tregs) are pivotal in the regulation of $\mathrm{T}$ cell-mediated immune responses in atherosclerosis, a chronic autoimmune-like disease. In the authors' previous studies, it was demonstrated that amygdalin ameliorated atherosclerosis by the regulation of Tregs in apolipoprotein E-deficient $\left(\mathrm{ApoE}^{-/-}\right)$mice. Therefore, the aim of the present study was to investigate the therapeutic effect of amygdalin on low-density lipoprotein (LDL) receptor deficient $\left(\mathrm{LDLR}^{-/}\right)$mice, and to examine its immune regulatory function by the stimulation of Tregs. To establish an atherosclerosis mouse model, the $\mathrm{LDLR}^{-/-}$mice were fed a high fat and high cholesterol diet then the total plasma cholesterol, triglyceride, LDL and chemokines levels were measured by an ELISA. Following sacrificing the mice, the upper sections of the aorta were stained by hematoxylin and eosin, and Oil red $\mathrm{O}$ to assess the plaque area. Then western blotting and reverse transcription polymerase
\end{abstract}

Correspondence to: Dr Yi Wang, Department of Pharmacy, Sichuan Academy of Medical Science and Sichuan Provincial People's Hospital, 2 West First Ring Road, Chengdu, Sichuan 610072, P.R. China

E-mail:w_yi@yahoo.com

Dr Jiagang Deng, Guangxi Key Laboratory of Efficacy Study on Chinese Materia Medica, Guangxi University of Chinese Medicine, 13 Wuhe Avenue, Nanning, Guangxi 530200, P.R. China

E-mail: dengjg53@126.com

${ }^{*}$ Contributed equally

Key words: amygdalin, atherosclerosis, matrix metalloproteinase-2, matrix metalloproteinase-9, monocyte chemoattractant protein-1, regulatory $\mathrm{T}$ cells chain reactions were performed to analysis the expression levels of cluster of differentiation 68, monocyte chemoattractant protein-1, matrix metalloproteinase (MMP)-2, MMP-9 and forkhead box P3 (Foxp3). To further confirm the activation of FOXP3 by amygdalin, lentiviruses carrying Foxp3 shRNA were injected into the mice, and the serum cytokines levels were measured by ELISA. Following feeding of the mice with a high-fat/high-cholesterol diet, the $\mathrm{LDLR}^{-/-}$mice demonstrated comparatively higher levels of triglyceride, total cholesterol and LDL, compared with levels in the amygdalin-treated mice. By comparing the vessel area, lumen area, plaque area, and percentage aortic plaque coverage, the effects of amygdalin on pre-existing lesions were assessed. In addition, the levels of CD68, monocyte chemoattractant protein-1, MMP-2 and MMP-9 were analyzed, and analysis of the expression of interleukin (IL)-1 $\beta$, IL- 6 and tumor necrosis factor (TNF)- $\alpha$ indicated that the mice treated with amygdalin had decreased expression of pro-inflammatory cytokines. The mRNA and protein levels of Foxp3 were also quantified, and the mice treated with amygdalin demonstrated an increased number of Tregs. The knockdown of Foxp3mRNA resulted in the increased secretion of IL-1 $\beta$, IL- 6 and TNF- $\alpha$. Therefore, the data indicated that amygdalin regulated the formation of atherosclerosis and stabilized the plaque by suppressing inflammatory responses and promoting the immune-modulation function of Tregs. Taken together, the results demonstrated the therapeutic effect of amygdalin on atherosclerosis.

\section{Introduction}

It is widely acknowledged that atherosclerosis is the principal cause of cardiovascular disease, which ultimately contributes to mortality rates $(1,2)$. Although hypercholesterolemia, smoking, male gender, hypertension, diabetes and age are traditionally considered to be risk factors, accumulating evidence indicates that hyperlipidemia is the major risk factor underlying the formation and development of atherosclerosis $(3,4)$. During the 
initiation of atherosclerosis, plasma low-density lipoprotein (LDL) accumulates in the arterial wall, oxidized, and recruits circulating monocytes (5). When monocytes transform into macrophages, they uptake oxidized (ox) LDL, resulting in substantial cholesterol accumulation and the formation of foam cells, which ultimately lead to formation of the atherosclerotic lesion. In this multifactorial process, endothelial cells, smooth muscle cells, macrophages and lymphocytes are involved and interact. Increasing experimental evidence suggests that during this process, an array of cytokines and adhesion molecules are involved and promote atherogenesis $(6,7)$. The innate and adaptive immune responses are also pivotal in the pathogenesis of atherosclerosis (8). Therefore, as a result of the multiple interactions, atherosclerotic plaques are formed, which ultimately results in cerebrovascular events and acute coronary syndromes.

Amygdalin (vitamin B17, also known as Laetrile), is extracted from Semen Persicae, the seed of Prunus Persica (L.) Batsch. Amygdalin is also found in abundance in the seeds of apricots, almonds, peaches and other rosaceous plants. To date, amygdalin has been widely used for the treatment of asthma, bronchitis, emphysema, leprosy, diabetes and cancer. The authors' previous study on amygdalin suggested that it may stimulate the immune system and exhibits immune modulation functions by the regulation of regulatory $\mathrm{T}$ cells (Tregs) in apolipoprotein E-deficient $(\mathrm{ApoE})^{-/-}$mice. As $\mathrm{ApoE}^{-/}$mice are spontaneous atherosclerotic mice, in order to determine whether amygdalin can alleviate high-fat/high-cholesterol induced atherosclerosis, $\mathrm{LDLR}^{-/-}$mice were used in the present study. It has been demonstrated that the cytokines produced by $T$ cells, including interleukin (IL)- 6 and tumor necrosis factor (TNF)- $\alpha$, and macrophages, including IL-1 $\beta$, affect the extent and nature of the atherosclerotic plaque $(9,10)$. The authors' previous study on the anti-atherosclerotic effect of amygdalin demonstrated that the expression levels of IL-10 and transforming growth factor (TGF)- $\beta$ were markedly increased in the serum of the amygdalin-treated mice. Therefore, it was hypothesized that amygdalin possesses a broader immune-regulative function on atherogenesis and is critical in the regulation of Tregs in high-fat/high-cholesterol diet-induced atherosclerotic $\mathrm{LDLR}^{-/-}$mice. To confirm this hypothesis, the present study aimed to determine whether the expression levels of TNF- $\alpha$, IL- $1 \beta$ and IL- 6 can be regulated by amygdalin. It is well known that Tregs have a protective role in the progression of atherosclerosis (11) and they are considered to be a therapeutic target for the treatment of atherosclerosis. The authors' previous studies suggested that Tregs can be induced and expanded by amgydalin (12). Therefore, to further substantiate the hypothesis, the expression of forkhead box P3 (Foxp3) was silenced through Foxp3-specific short hairpin (sh)RNAs and the expression levels of the cytokines were observed. Previous clinical investigations of matrix metalloproteinase (MMP)-2 and MMP-9 demonstrated that MMP-9 activity was found in macrophage-rich lesions, whereas the expression of MMP-2 was higher in lesions rich in smooth muscle cells, indicating that MMP-2 and MMP-9 are tightly associated with stable and vulnerable lesions (13). In order to elucidate the mechanism underlying the anti-atherosclerotic function of amygdalin, the present study also examined the expression levels of MMP-2 and MMP-9. Therefore, in order to determine whether amygdalin treatment attenuates plaque progression and alleviates the symptom of atherosclerosis, the present study focused on the immune regulatory function of amygdalin.

\section{Materials and methods}

Animals. The $\mathrm{LDLR}^{-/-}$mice were purchased from Jackson Laboratories (Bar Harbor, ME, USA) and maintained in a 12-h light/dark cycle in an atmosphere of $0.03 \% \mathrm{CO}_{2}$ with free access to water and food. In accordance with the individual ventilated cage requirements of Sichuan Academy of Medical Science \& Sichuan Provincial People's Hospital (Sichuan, China), all mice were raised under specific pathogen-free conditions with controlled temperature $\left(23 \pm 2^{\circ} \mathrm{C}\right)$. The use and handling of animals were in accordance with the Ethics Committee of Sichuan Academy of Medical Science \& Sichuan Provincial People's Hospital.

Study design. Amygdalin was purchased from Changsha Staherb Natural Ingredients Co., Ltd. (Changsha, Hunan, China). The 8 -week-old male mice were divided into five groups ( $\mathrm{n}=30$ in each group, and body weight range of $\sim 22-24$ g): i) LDLR $^{-/}$control group (control): 8-week-old male $\mathrm{LDLR}^{-/}$mice were fed a standard laboratory diet (10\% fat, $15 \%$ protein and $75 \%$ carbohydrate); ii) $\mathrm{LDLR}^{-/}$high-fat diet (HFD) control group (LDLR ${ }^{-1}$ ): 8-week-old male $\mathrm{LDLR}^{-1-}$ mice were fed an HFD containing $40 \%$ fat, $14 \%$ protein and $46 \%$ carbohydrate for 12 weeks; iii) Amygdalin (Low) group: 8 -week-old male $\mathrm{LDLR}^{-/}$mice were fed the HFD for 12 weeks and amygdalin (1 mg/kg/day for 4 weeks consecutively) was injected intraperitoneally; iv) Amygdalin (medium) group: 8-week-old male $\mathrm{LDLR}^{-/}$mice were fed the HFD for 12 weeks and amygdalin ( $3 \mathrm{mg} / \mathrm{kg} / \mathrm{day}$ for 4 weeks consecutively) was injected intraperitoneally; v) Amygdalin (high) group: 8-week-old male $\mathrm{LDLR}^{-/}$mice were fed the HFD for 12 weeks and amygdalin $(10 \mathrm{mg} / \mathrm{kg} / \mathrm{day}$ for 4 weeks consecutively) was injected intraperitoneally. All groups of mice were sacrificed following 4 weeks of drug delivery.

Lipid profile and cytokine measurements. For measuring total cholesterol content, blood samples were collected from each mouse at the beginning and end of amygdalin treatment. Total plasma cholesterol (TC) and triglyceride (TG) levels were determined using commercial kits (Applygen Technologies, Inc., Beijing, China). The levels of murine IL-1 $\beta$, TGF- $\alpha$ and IL-6 were assayed using ELISA kits with paired antibodies according to the manufacturer's protocol (R\&D Systems, Inc. Minneapolis, MN, USA).

Assessment of aortic sinus atherosclerosis. The hearts and upper sections of the aorta were removed from the mice, fixed, embedded in paraffin and sectioned $(5-\mu \mathrm{m})$. The sections were then stained with hematoxylin and eosin (cat. no. C0105; Beyotime Institute of Biotechnology, Suzhou, China) and oil red O (cat. no. O0625; Sigma-Aldrich; Merck KGaA, Darmstadt, Germany). Atherosclerotic lesions were quantified by calculating the lesion size in the aortic sinus, as previous described $(14,15)$. The vessel areas were measured using ImageJ software (version 1.47; National Institutes of 
Health; Bethesda, MD, USA) from images obtained with a Nikon 80i microscope (Nikon Corporation, Tokyo, Japan). For comparisons of plaque areas between the amygdalin groups and control groups, 100 and $200 \mu \mathrm{m}$ distant sites were used.

Western blot analysis. The proteins were lysed by radioimmunoprecipitation lysis buffer (Beyotime Institute of Biotechnology) and quantified with bicinchoninic method (Beyotime Institute of Biotechnology) as previously described (12). A total of $20 \mu \mathrm{g}$ protein was loaded onto a NuPageNovex 10\% Bis-Tris gel (Thermo Fisher Scientific, Inc.) for electrophoresis. Following electrophoresis, the proteins were transferred onto polyvinylidene fluoride membranes (Pall Life Sciences, Port Washington, NY, USA). The membranes were blocked bovine serum albumin (Sigma-Aldrich; Merck $\mathrm{KGaA}$ ), and then incubated with primary antibodies overnight at $4^{\circ} \mathrm{C}$ followed by incubation with horseradish peroxidase (HRP)-conjugated secondary antibody at room temperature for $2 \mathrm{~h}$. Chemiluminescence detection was performed using Immobilon Western Chemiluminescent HRP substrate (EMD Millipore, Billerica, MA, USA), and measured directly using a BioSpectrum imaging system (UVP, Inc., Upland, CA, USA). The mouse monoclonal CD68 (cat. no. sc-20060; 1:1,000), mouse monoclonal MMP-2 (cat. no. sc-13594; 1:500), mouse monoclonal MMP-9 (cat. no. sc-393859; 1:500) and mouse monoclonal $\beta$-actin (cat. no. sc-47778; 1:5,000) were purchased from Santa Cruz Biotechnology (Dallas, TX, USA). Rabbit polyclonal monocyte chemoattractant protein-1 (MCP-1; cat. no. ab9899; 1:500) was purchased from Abcam (Cambridge, MA, USA). T cells from the spleen were isolated from the mice in each group. Western blot analysis was performed with mouse monoclonal anti-Foxp3 antibody (cat. no. ab20034; 1:500; Abcam). For the secondary antibody, HRP-conjugated goat anti-mouse IgG polyclonal antibody (cat. no. 115-035-003) and HRP-conjugated goat anti-rabbit polyclonal IgG (cat. no. 111-035-003) were used, which were purchased from Jackson ImmunoResearch Laboratories, Inc. (West Grove, PA, USA). The levels of $\beta$-actin were determined as the loading control.

Reverse transcription-quantitative polymerase chain reaction $(R T-q P C R)$ analysis. Total RNA from the splenocytes and lymphocyte cells was isolated using TRIzol reagent (Invitrogen; Thermo Fisher Scientific, Inc.). The primer sequences: FOXP3 forward, 5'-CCCAGGAAAGACAGC AACCTT-3' and reverse, 5'-TTCTCACAACCAGGCCAC TTG-3'; MCP-1 forward, 5'-TTAAAAACCTGGATCGGA ACCAA-3' and reverse, 5'-GCATTAGCTTCAGATTTA CGGG-3'; MMP-2 forward, 5'-ACCCAGATGTGGCCAACT AC-3' and reverse, 5'-TACTTTTAAGGCCCGAGCAA-3'; and MMP-9 forward, 5'-ATGATGGAGGAGAAGCAGTC-3' and reverse, 5'-AGGTGAAGGGAAAGTGACAT-3' were used as previously described $(12,16,17)$. The RT-qPCR analysis was performed on an ABI 7900 system using Applied Biosystems Power SYBR ${ }^{\circledR}$ Green PCR Master mix (Thermo Fisher Scientific, Inc.) in triplicate (18). In brief, $0.5 \mu \mathrm{M}$ forward and reverse primes, $2 \mu \mathrm{l}$ buffer and $100 \mathrm{ng}$ cDNA templates were added to each tube and the total volume was adjusted to $20 \mu \mathrm{l}$ by RNase free water (Thermo Fisher Scientific Inc.). Following 3 min denaturation at $95^{\circ} \mathrm{C}$; amplification was performed for
35 cycles, including $95^{\circ} \mathrm{C}$ for $10 \mathrm{sec}$ for denaturation and $55^{\circ} \mathrm{C}$ for $30 \mathrm{sec}$ for annealing and extension. The copy number was calculated using the $2^{-\Delta \Delta \mathrm{Cq}}$ method. The $\mathrm{Cq}$ value for GAPDH was used to normalize the samples (19).

Lentivirus preparation and injection. The Foxp3 target sequences were as follows: forward, 5'-GGACACUCAAUG AAAUCUATT-3'; reverse, 5'-UAGAUUUCAUUGAGUGUC CTC-3' (20). The shRNAs were synthesized and cloned into pTY linkers. Third-generation vectors were used in this experiment. The shRNA lentiviral vector was transiently transfected into 293T cells (Type Culture Collection of the Chinese Academy of Sciences, Shanghai, China). Briefly, the 293T cells were co-transfected with appropriate quantities of vector plasmids, including the helper construct, envelope plasmid, tat plasmid and pTYlinker containing shRNAs. The viral supernatant was harvested at $48 \mathrm{~h}$, filtered through a $0.45-\mu \mathrm{m}$ filter, subjected to ultracentrifugation $\left(113,000 \mathrm{x} \mathrm{g}\right.$ at $4^{\circ} \mathrm{C}$ for $\left.2 \mathrm{~h}\right)$ for 100 -fold concentration and stored at $-80^{\circ} \mathrm{C}$. Then lentiviral supernatant was thawed at $37^{\circ} \mathrm{C}$ and diluted in $0.9 \%$ saline (Sichuan Kelun Pharmaceuticals, Co., Ltd., Chengdu, Sichuan, China) and polybrene $(8 \mu \mathrm{g} / \mathrm{ml}$ final concentration; Sigma-Aldrich; Merck $\mathrm{KGaA}$ ) to give a dose of $1.6 \times 10^{7}$ or $1.6 \times 10^{8}$ transducing units in a $50 \mu \mathrm{l}$ injection volume. The virus was injected intravenously into the mice with a 30-gauge needle on a 1-cc syringe. After the $\mathrm{LDLR}^{-/-}$mice were confirmed as atherosclerotic, 3 consecutive injections were administered at 3-day intervals $(n=6)$.

Statistical analysis. The effects of treatment on the lesions, plaque compositions, levels of IL-1 $\beta$, IL- 6 and TNF- $\alpha$, and mRNA levels were calculated using one-way analysis of variance and Bonferroni/Dunn's test or Student's t-test by GraphPad Prism $^{\mathrm{TM}}$ software (version 5.0; GraphPad Software Inc., La Jolla, CA, USA). $\mathrm{P}<0.05$ was considered to indicate a statistically significant difference.

\section{Results}

Amygdalin regulates lipid composition and the development of atherosclerosis. The authors' previous data indicated that amygdalin attenuated atherosclerosis in $\mathrm{ApoE}^{-/-}$mice. To further examine the therapeutic effect of amygdalin in atherosclerosis, an atherosclerotic mouse model was established in the present study by feeding LDLR $^{-/}$mice with an HFD. It was evident from analyzing the lipid profile (Table I), that the TG levels and TC levels were increased in the $\mathrm{LDLR}^{-/}$mice. However, these changes were alleviated when the mice were treated with amygdalin. A high dose of amygdalin resulted in the 2-fold decrease in TG levels, compared with those in the LDLR $^{-/-}$mice $(\mathrm{P}<0.05)$. By comparing the TC and LDL levels between the drug delivery groups and corresponding control groups, it was found that the mice treated with amygdalin had decreased levels of TC and LDL $(\mathrm{P}<0.05)$. In addition, increased levels of HDL were observed in the amygdalin-treated mice $(\mathrm{P}>0.05)$. Therefore, amygdalin regulated lipid contents in the HFD-treated LDLR $^{-/-}$mice.

As the atherosclerotic lesions in the $\mathrm{LDLR}^{-/-}$mice fed with the HFD demonstrated the same morphological characteristics as those of humans, and the mouse model imitated the 
Table I. Lipid profile of different groups of mice ( $\mathrm{n}=10$ in each group).

\begin{tabular}{lcccc}
\hline Group & TG (mmol/l) & TC (mmol/l) & HDL (mmol/l) & LDL (mmol/l) \\
\hline Control & $1.05 \pm 0.14^{\mathrm{a}}$ & $1.62 \pm 0.43^{\mathrm{a}}$ & $0.75 \pm 0.04$ & $0.82 \pm 0.29^{\mathrm{a}}$ \\
LDLR $^{-/}$ & $4.96 \pm 1.21$ & $4.13 \pm 2.73$ & $0.72 \pm 0.13$ & $5.42 \pm 0.36$ \\
Amygdalin (low) & $3.71 \pm 0.51^{\mathrm{a}}$ & $3.62 \pm 0.38^{\mathrm{a}}$ & $0.95 \pm 0.08^{\mathrm{a}}$ & $4.56 \pm 0.26^{\mathrm{a}}$ \\
Amygdalin (medium) & $2.18 \pm 0.48^{\mathrm{a}}$ & $3.36 \pm 0.26^{\mathrm{a}}$ & $1.27 \pm 0.11^{\mathrm{a}}$ & $3.65 \pm 0.30^{\mathrm{a}}$ \\
Amygdalin (high) & $1.75 \pm 0.33^{\mathrm{a}}$ & $2.61 \pm 0.58^{\mathrm{a}}$ & $1.55 \pm 0.25^{\mathrm{a}}$ & $2.74 \pm 0.27^{\mathrm{a}}$ \\
\hline
\end{tabular}

TG, triglyceride; TC, total cholesterol; HDL, high density lipoprotein; LDL, low density lipoprotein; LDLR ${ }^{-/}$, low-density lipoprotein receptor deficiency. ${ }^{\text {aP }}<0.05$ vs. LDLR $^{-1}$ group.

progression of atherosclerosis, the aortic sinus was stained with H\&E and oil red $\mathrm{O}$ in the present study (Fig. 1A). The atherosclerotic plaques were well developed in the $\mathrm{LDLR}^{-1}$ mice. By contrast, no atherosclerotic plaques were found in the amygdalin-treated mice. The, vessel areas, lumen areas and plaque areas were also analyzed. As exhibited in Fig. 1B, with the progression of atherosclerosis, the diameters of the aortic sinus increased. In addition, the areas of the lumen were decreased (Fig. 1C) and those of the plaques were increased (Fig. 1D) in the LDLR $^{-/}$mice. By contrast, the mice treated with amygdalin exhibited a significantly reduced extent of atherosclerosis, evident by the smaller aortic sinus plaques, smaller aortic sinus and expanded lumen are as. This finding was confirmed by the analysis of atherosclerosis surface coverage areas (Fig. 1E), showing decreased lesions in the amygdalin-treated LDLR $^{-/-}$mice. Therefore, these data indicated that amygdalin inhibited the HFD-induced increase in atherosclerotic lesions in the $\mathrm{LDLR}^{-/}$mice.

Amygdalin inhibits the inflammatory response. To investigate whether amygdalin inhibits inflammation in the aorta of $\mathrm{LDLR}^{-/}$mice, the present study compared the mRNA and protein levels of MCP-1, CD68, MMP-2 and MMP-9 in the atherosclerotic arteries. As shown in Fig. 2A and B, the LDLR $^{-/}$mice exhibited a marked increase in the expression levels of CD68, MCP-1, MMP-2 and MMP-9. As expected, decreased activities of CD68, MCP-1, MMP-2 and MMP-9 were observed following amygdalin treatment. In addition, to further confirm the inhibitory function of amygdalin in inflammation, plasma inflammatory markers were assessed. As predicted, the analysis of serum inflammatory cytokines revealed that amygdalin markedly reduced the secretion of IL-1 $\beta$ (Fig. 3A), IL-6 (Fig. 3B), and TNF- $\alpha$ (Fig. 3C).

Amygdalin inhibits the progression of atherosclerosis via the induction of Tregs. To determine whether the presence of Tregs is associated with lipid profile changes and inhibited inflammatory responses following amygdalin delivery, the present study investigated the mRNA and protein levels of Foxp3 in splenocytes and peripheral blood cells. Following quantification of them RNA levels of Foxp3, the mRNA levels in peripheral blood cells were increased by 6.3 -fold (Fig. 4A), and the mRNA levels in splenocytes were increased by 3.5 -fold (Fig. 4B) in the groups treated with a high-dose of amygdalin, compared with the untreated groups $(\mathrm{P}<0.05)$.
Even in the groups treated with a low dose of amygdalin, the mRNA levels of Foxp3 in the peripheral blood cells and splenocytes were markedly elevated. There was also a marked increase in the protein levels of Foxp3in the amygdalin-treated groups (Fig. 4C).

A previous report indicated that the deletion of Foxp3 results in the absence of functional Tregs in the periphery and a conditionally active form of Foxp3 induces the reprogramming of human T cells into Tregs (21). To further establish that Tregs are induced by amygdalin, the knockdown of Foxp3mRNA was achieved using a lentiviral vector carrying shRNA (Fig. 5A), following which serum proinflammatory cytokines were assessed. As expected, the levels of IL-1 $\beta$ (Fig. 5B), IL-6 (Fig. 5C) and TNF- $\alpha$ (Fig. 5D) were significantly increased in the amygdalin-treated mice bearing Foxp3 shRNA. These data indicated that amygdalin attenuated the development of atherosclerosis via the regulation of Tregs and proinflammatory cytokines in $\mathrm{LDLR}^{-/}$mice.

\section{Discussion}

The authors' previous studies on amygdalin in $\mathrm{ApoE}^{-/}$mice demonstrated that amygdalin ameliorated the progression of atherosclerosis by the regulation of Tregs (12). However, to assess the regulatory role of amygdalin on atherosclerosis induced by a western diet, the $\mathrm{LDLR}^{-/-}$mouse model was used in the present study. Unlike $\mathrm{ApoE}^{-/-}$mice, a spontaneous atherosclerotic model, LDLR $^{-/}$mice do not develop atherosclerosis if they are not fed with an HFD. A previous study demonstrated that the oral administration of oxLDL induced attenuated atherosclerosis in $\mathrm{LDL}^{-1-}$ mice (22), indicating that the abnormal lipid composition is involved in the progression of atherosclerosis. The present study demonstrated that amygdalin significantly inhibited the development of atherosclerosis in LDLR $^{-/}$male mice fed an HFD as a western-style diet. These mice, in addition to exhibiting hypercholesterolemia, exhibited hypertriglyceridemia, therefore, they exhibited the clinical features of human hypercholesterolemia and hypertriglyceridemia. On comparing the blood cholesterol levels, blood lipids were significantly decreased following amygdalin treatment. This observation was consistent with our previous study in $\mathrm{ApoE}^{-/}$mice, indicating that amygdalin is critical in the regulation of lipid profiles. Of note, in the present study, the HFD produced more advanced atherosclerotic lesions in the $\mathrm{LDLR}^{-/-}$mice. 
A

H\&E staining

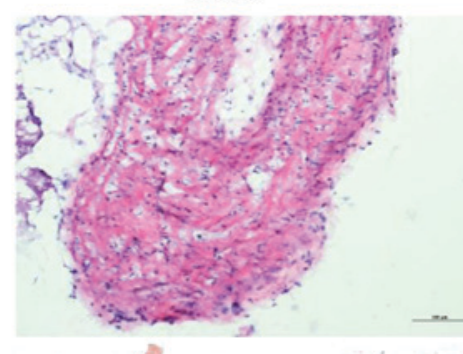

Oil red $\mathrm{O}$ staining

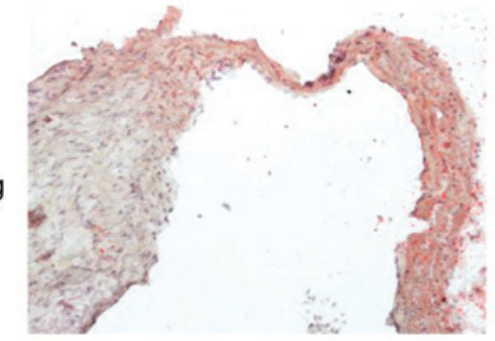

B

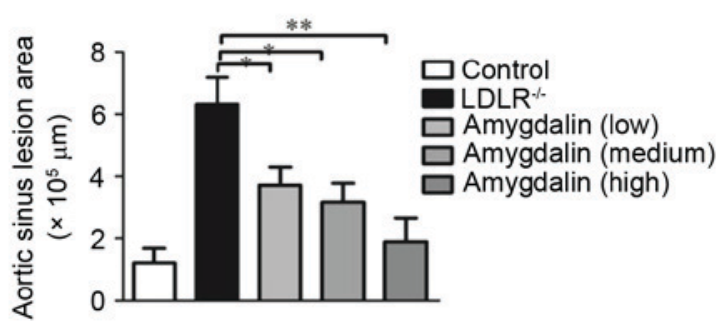

D

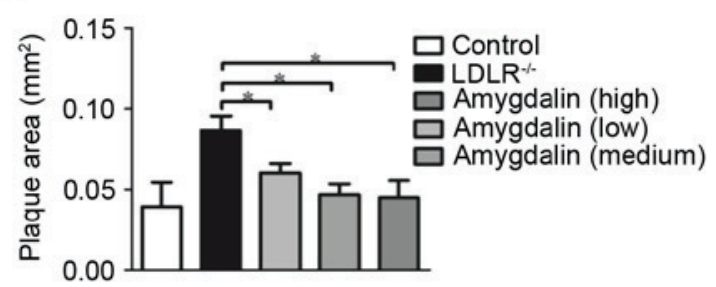

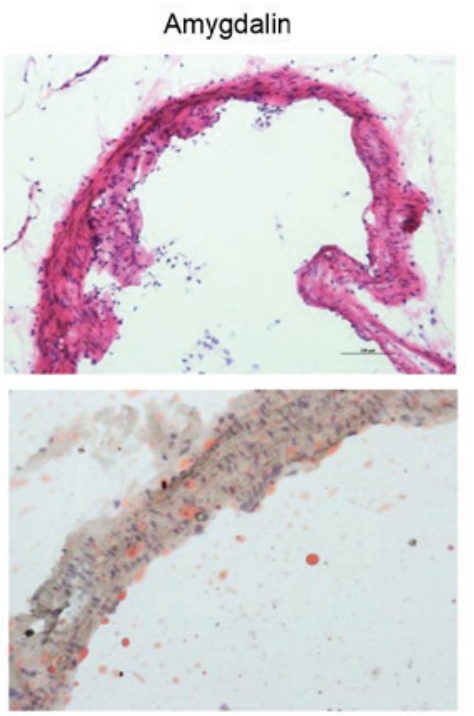

C

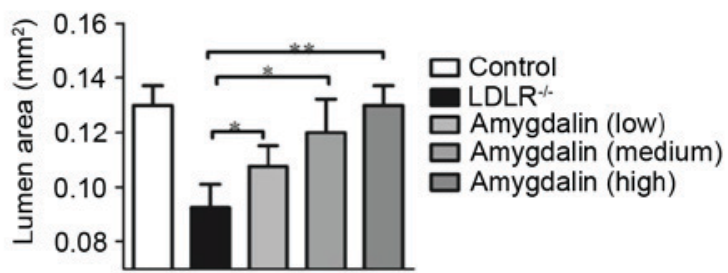

E

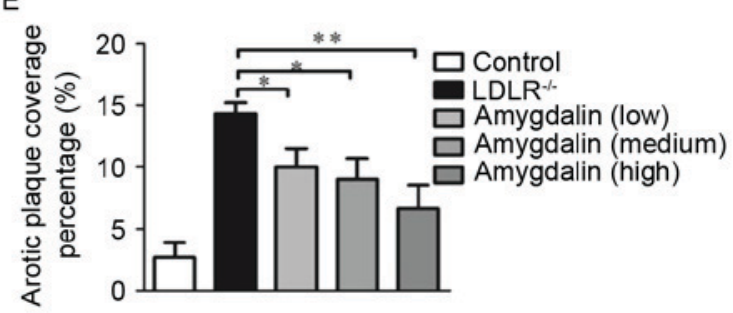

Figure 1. $\mathrm{LDLR}^{-/}$mice treated with amygdalin reduce signs of atherosclerotic plaques. (A) Representative images of $\mathrm{H} \& \mathrm{E}$ staining and oil red $\mathrm{O}$ staining of mice aorta tissues (magnification, x100). Graphs show results of statistical analyses of (B) aortic sinus lesion area, (C) lumen area, (D) plaque area, and (E) percentage aortic plaque coverage. Amygdalin treatment significantly reduced the signs of atherosclerosis. Data are presented as the mean \pm standard error of the mean. ${ }^{*} \mathrm{P}<0.05$ and $^{* * *} \mathrm{P}<0.01$. LDLR ${ }^{-1}$, low-density lipoprotein receptor deficiency; H\&E, hematoxylin and eosin.

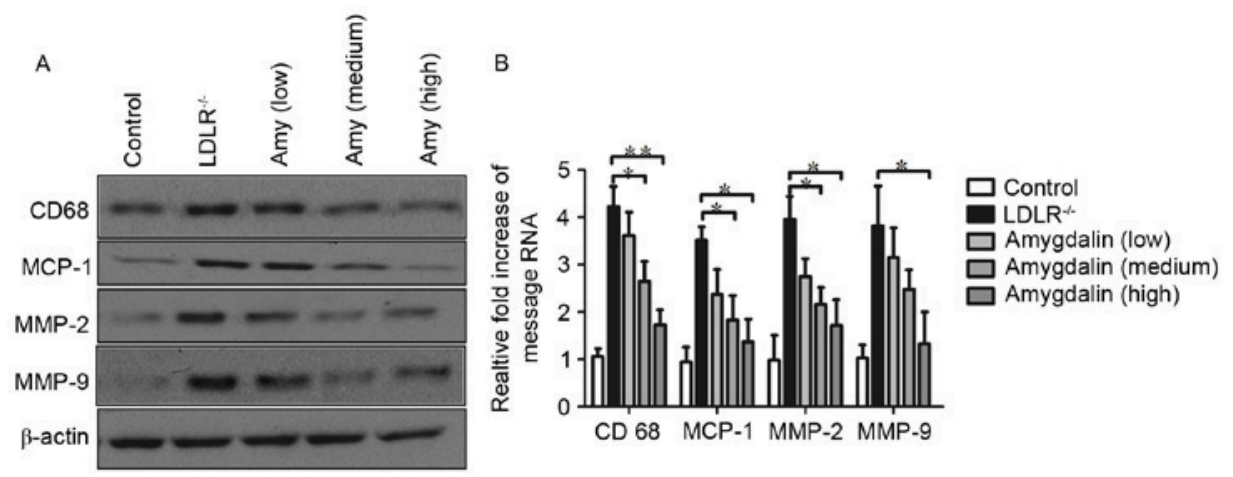

Figure 2. Protein expression levels of CD68, MCP-1, MMP-2 and MMP-9. (A) Western blot analysis demonstrated that amygdalin treatment reduced the protein levels of CD68, MCP-1, MMP-2 and MMP-9. (B) Relative mRNA levels were quantified. Data are presented as the mean \pm standard error of the mean from three independent experiments. $\mathrm{P}<0.05$ and ${ }^{* * *} \mathrm{P}<0.01$. $\mathrm{LDLR}^{-1}$, low-density lipoprotein receptor deficiency; MCP-1, monocyte chemoattractant protein-1; MMP, matrix metalloproteinase.

There is increasing evidence that cytokines produced by $\mathrm{CD} 4^{+} \mathrm{T}$ cells and macrophages, including TNF- $\alpha$, IL- $1 \beta$ and IL-6, affect the extent and nature of the atherosclerotic plaque. A substantial number of clinical studies have 
A

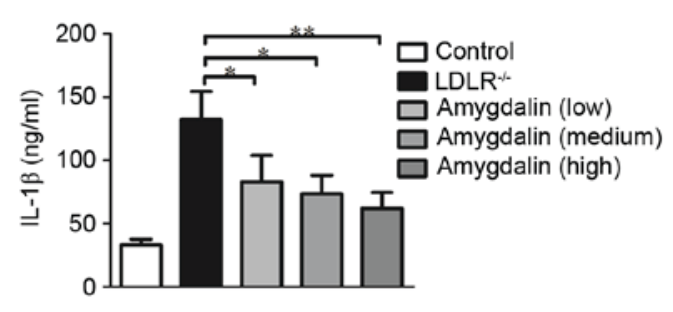

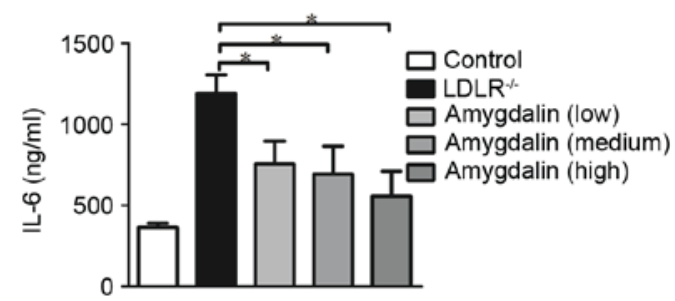

C

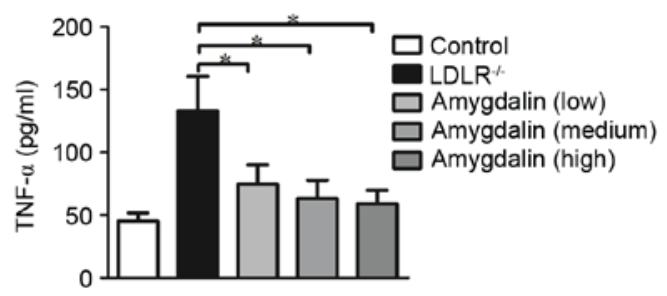

Figure 3. Amygdalin inhibits serum cytokine secretion. Serum levels of (A) IL-1 $\beta$ (B) IL-6 and (C) TNF- $\alpha$ were analyzed. Data are presented as the mean \pm standard error of the mean from three independent experiments. ${ }^{*} \mathrm{P}<0.05$ and ${ }^{* *} \mathrm{P}<0.01$. IL, interleukin; TNF- $\alpha$, tumor necrosis factor- $\alpha$; LDLR ${ }^{-/}$, low-density lipoprotein receptor deficiency.

A

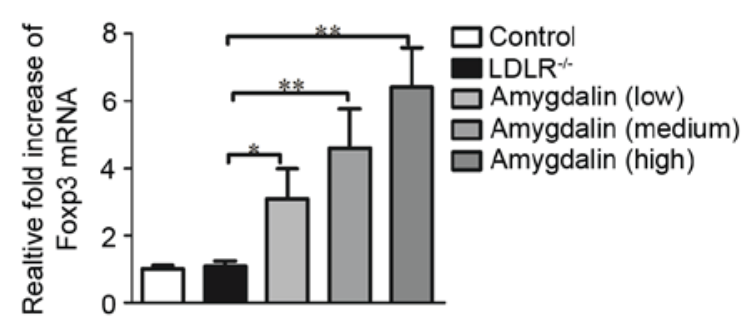

B

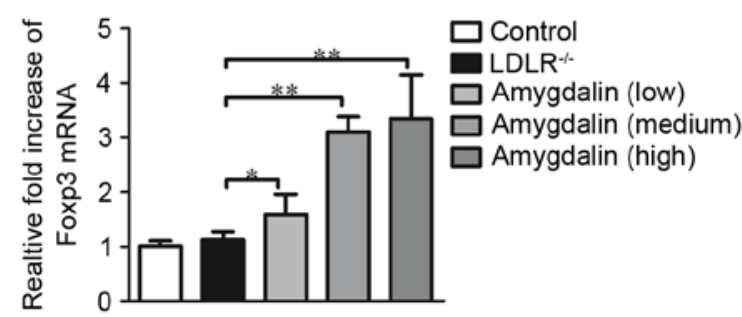

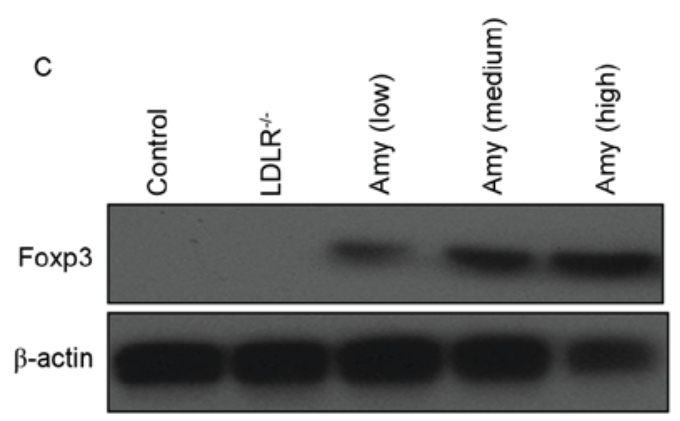

Figure 4. Amygdalin induces Tregs in LDLR ${ }^{-/}$mice. mRNA levels of Foxp3 in the (A) blood and (B) splenocytes were analyzed. (C) Protein levels of Foxp3 were quantified using western blot analysis. Data are presented as the mean \pm standard error of the mean from three independent experiments. $\mathrm{P}<0.05$ and ${ }^{* *} \mathrm{P}<0.01$. LDLR ${ }^{-/}$, low-density lipoprotein receptor deficiency; Amy, amygdalin; Foxp3, forkhead box P3.

shown that the plasma concentration of TNF- $\alpha$ is associated with the degree of atherosclerosis $(23,24)$. To date, epidemiological studies have found that several inflammatory mediators, including IL- 6 and TNF- $\alpha$, appear to be elevated in association with increased vascular risk, indicating cytokine-mediated inflammation is present in the early stages of atherogenesis $(25,26)$. Huber et al demonstrated that the injection of recombinant IL-6 exacerbated development of the early lesion of atherosclerosis in mice (27). Studies on IL-1 $\beta$ have also indicated that the overexpression of IL-1 $\beta$ occurs in various inflammatory diseases, including atherosclerosis, and a reduction of IL- $1 \beta$ decreases the severity of atherosclerosis in $\mathrm{ApoE}^{-/-}$mice $(28,29)$. Studies by Kirii et al demonstrated a significant decrease in atherosclerotic lesions at the aortic sinus in $\mathrm{ApoE}^{-/ /} / \mathrm{IL}^{-1} \beta^{-/}$mice, and the mRNA levels of MCP-1 in the aorta were improved in these mice (28). Their results indicated that the reduction of IL-1 $\beta$ decreases the severity of atherosclerosis through increasing the expression of MCP-1. It has also been reported that macrophage foam cells and smooth muscle cells express IL-6, indicating that IL- 6 , in addition to IL- $1 \beta$ and TNF- $\alpha$, are critical in the progression of atherosclerosis (9). In the present study, it was demonstrated that even a low dose of amygdalin markedly reduces the expression levels of circulating cytokines, including IL-6, IL-1 $\beta$ and TNF- $\alpha$. However, compared with the low dose group, no significant 
A

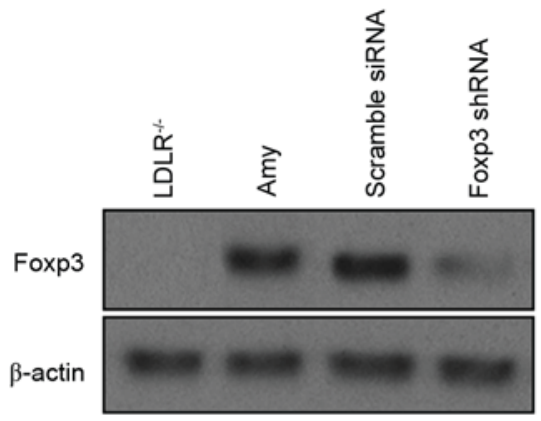

C

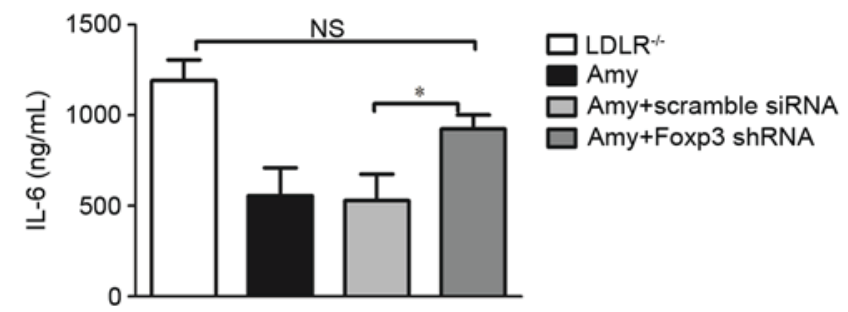

B

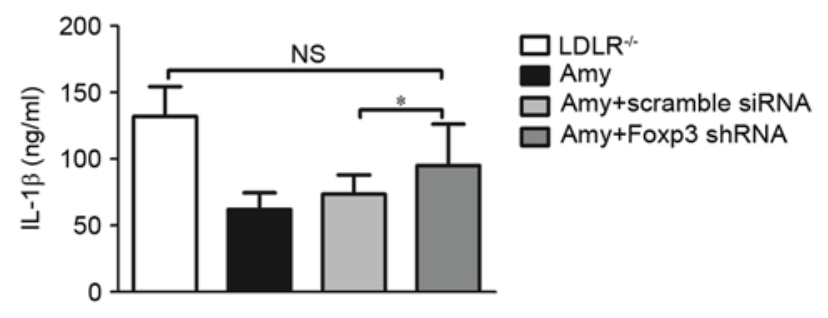

$\mathrm{D}$

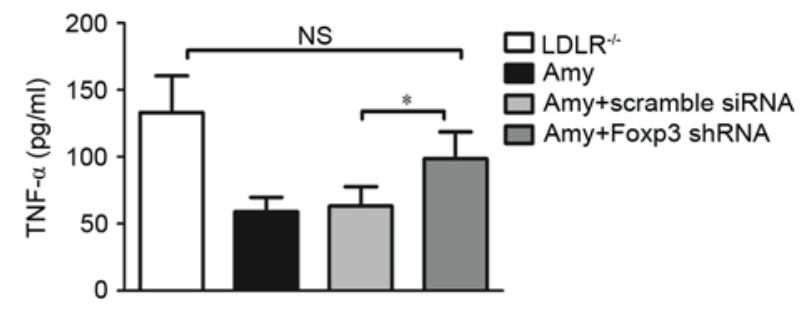

Figure 5. Silencing of Foxp3 results in increased pro-inflammatory cytokine secretion. (A) Representative images of western blots following Foxp3 knockdown. Levels of Serum (B) IL-1 $\beta$, (C) IL-6 and (D) TNF- $\alpha$ were analyzed. Data are presented as the mean \pm standard error of the mean from three independent experiments. " $\mathrm{P}<0.05$. IL, interleukin; TNF- $\alpha$, tumor necrosis factor- $\alpha$; LDLR $^{--}$, low-density lipoprotein receptor deficiency; siRNA, small interfering RNA; shRNA, short hairpin RNA; Amy, amygdalin; NS, no significant difference.

difference in the reduction of proinflammatory cytokines was observed in when the mice were treated with a high dose of amygdalin.

It is widely acknowledged that Tregs are important in the development of atherosclerosis, confirmed by studies investigating the adoptive transfer of lymphocytes $(15,30)$ and Th2 cytokines produced by Tregs. In the authors' previous study, the anti-atherosclerotic function of amygdalin was demonstrated by its successful induction of Tregs in $\mathrm{ApoE}^{-/-}$mice. However, in the high-fat/high-cholesterol diet-induced atherosclerosis of $\mathrm{LDLR}^{-/}$mice, whether the anti-atherosclerotic effect of amygdalin is via the regulation of Tregs remains to be elucidated. Foxp3 has been demonstrated to govern the development and function of Tregs (31). Mutations in Foxp3 eliminate CD25 ${ }^{+}$ Tregs and cause autoimmune disorders $(32,33)$. Therefore, the present study also focused on Tregs, and further substantiation of our hypothesis was achieved by the silencing of Foxp3. As expected, elevated mRNA and protein expression levels of Foxp3 were observed in the amygdalin-treated mice. Following silencing of Foxp3, a marked increase in cytokines was observed in the circulation, indicating that the inhibition of Tregs exacerbated the atherosclerotic situation. These results provide evidence that the inhibition of Foxp3 acceleratedthe development of atherosclerosis, even with amygdalin treatment, suggesting that the anti-atherosclerotic effect of amygdalin occurred through the regulation of Tregs.

Although it is widely acknowledged that lipids are central in the pathogenesis of plaques, macrophages, and their secreted cytokines contribute substantially to atherogenesis. It has been reported that M1 macrophages in atheroma may have pro-inflammatory functions, which produce high levels of effectors, including cytokines IL-1 $\beta$ and TNF- $\alpha$ (13). According to the results of the present study, demonstrated in Fig. 3, amygdalin significantly reduced the cytokine secretion of M1 macrophages, suggesting the anti-atherosclerotic effect of amygdalin may be partly due to the inhibition of proinflammatory cytokines. Specifically, the mice treated with a high dose of amygdalin showed significantly decreased expression of CD68, indicating that the high dose of amygdalin exerted a more marked inhibitory effect on proinflammatory cytokines. As atheroma formation also involves the recruitment of $\mathrm{T}$ cells and chemokines, the present study examined other factors contributing to atherosclerosis. It has been documented that MCP-1 has a unique and crucial role in the initiation and evolution of atherosclerosis by regulating the migration of monocytes and T-cells into the vessel wall $(34,35)$. In the present study, an increased expression level of MCP-1 was observed in the atherosclerotic mice, however, treatment with amygdalin markedly reduced the chemokine secretion in the region of the lesion. The migration of SMCs also contributes to vascular remodeling during the development and complication of human atherosclerotic lesions. Clinical data show that MMP-2 facilitates the migration and proliferation of SMCs and may be important in atherogenesis (34). Cumulative evidence has demonstrated that MMP-2-deficiency reduces atherosclerotic plaque lesion formation in $\mathrm{ApoE}^{-/-}$mice (7). In clinical studies, that increased MMP-2 and MMP-9 staining was observed in the plaques of expansively remodeled segments, indicating that MMP-2 and MMP-9 may be involved in plaque vulnerability and in expansive arterial remodeling (36). By contrast, deficiency of MMP-9 has also been associated with reduced plaque size, macrophage content and collagen deposition in aortic lesions of $\mathrm{ApoE}^{-/-}$mice (37) and $\mathrm{LDLR}^{-/} / \mathrm{Apob}^{100 / 100}$ mice (19). In the present study, it was demonstrated that a high dose of amygdalin markedly reduced MMP-9 at the transcription and translation levels, indicating the anti-atherosclerotic effect of amygdalin via the inhibition of MMP-9. 
In conclusion, the present study demonstrated that, in the development of atherosclerosis in $\mathrm{LDLR}^{-/-}$mice fed with an HFD (western-style diet), amygdalin inhibited the progression of atherosclerosis. Amygdalin also markedly alleviated hypercholesterolemia and hypertriglyceridemia in the $\mathrm{LDLR}^{-/-}$mice and affected the extent of atherosclerotic lesions. Subsequent investigations on the mechanism of amygdalin-induced anti-atherosclerotic effects revealed that amygdalin inhibited the inflammatory response and induced Tregs. These results demonstrated the potential of amygdalin in the control and treatment of atherosclerosis, and support the potential of the clinical application of amygdalin as a novel anti-inflammatory drug in the prevention of coronary heart disease.

\section{Acknowledgements}

The present study was supported in part by the Guangxi Natural Science Foundation for Youth (grant no. 2013GXNSFBA019139), Guangxi Colleges and Universities Science and Technology Research Funding (grant no. ZD2014067), the Guangxi Health and Family Planning Commission Funding (grant no. GZPT13-05), the Guangxi Key Laboratory of Chinese Medicine Foundation Research Funding, Guangxi University of Chinese Medicine (grant no. 13-051-35) and Guangxi Key Laboratory of Common Technology of Traditional Chinese Medicine Preparation Funding, Guangxi University of Chinese Medicine to Dr Jianzhen Lv. The present study was also supported by the Sichuan Health and Family Planning Commission Funding (grant no. 16ZD0253), the Sichuan National Science Research Funding (grant no. 2015JY0183) and the Sichuan Scientific Research Foundation of the Returned Overseas Chinese Scholars to Dr Yi Wang.

\section{References}

1. Glass CK and Witztum JL: Atherosclerosis. The road ahead. Cell 104: 503-516, 2001.

2. Hansson GK: Inflammation, atherosclerosis, and coronary artery disease. N Engl J Med 352: 1685-1695, 2005.

3. Ross R and Harker L: Hyperlipidemia and atherosclerosis. Science 193: 1094-1100, 1976.

4. Tell GS, Crouse JR and Furberg CD: Relation between blood lipids, lipoproteins, and cerebrovascular atherosclerosis. A review. Stroke 19: 423-430, 1988.

5. Napoli C, D'Armiento FP, Mancini FP, Postiglione A, Witztum JL, Palumbo G and Palinski W: Fatty streak formation occurs in human fetal aortas and is greatly enhanced by maternal hypercholesterolemia. Intimal accumulation of low density lipoprotein and its oxidation precede monocyte recruitment into early atherosclerotic lesions. J Clin Invest 100: 2680-2690, 1997.

6. Ross R: Atherosclerosis-an inflammatory disease. N Engl J Med 340: 115-126, 1999.

7. Tedgui A and Mallat Z: Cytokines in atherosclerosis: Pathogenic and regulatory pathways. Physiol Rev 86: 515-581, 2006.

8. Binder CJ, Chang MK, Shaw PX, Miller YI, Hartvigsen K, Dewan A and Witztum JL: Innate and acquired immunity in atherogenesis. Nat Med 8: 1218-1226, 2002.

9. Whitman SC, Ravisankar P,Elam H and Daugherty A: Exogenous interferon-gamma enhances atherosclerosis in apolipoprotein E-/- mice. Am J Pathol 157: 1819-1824, 2000.

10. Frostegård J, Ulfgren AK, Nyberg P, Hedin U, Swedenborg J, Andersson $U$ and Hansson GK: Cytokine expression in advanced human atherosclerotic plaques: Dominance of pro-inflammatory (Th1) and macrophage-stimulating cytokines. Atherosclerosis 145: 33-43, 1999.
11. Mallat Z, Gojova A, Brun V, Esposito B, Fournier N, Cottrez F, Tedgui A and Groux H: Induction of a regulatory $\mathrm{T}$ cell type 1 response reduces the development of atherosclerosis in apolipoprotein E-knockout mice. Circulation 108: 1232-1237, 2003.

12. Jiagang D, Li C, Wang H, Hao E, Du Z, Bao C, Lv J and Wang Y: Amygdalin mediates relieved atherosclerosis in apolipoprotein E deficient mice through the induction of regulatory T cells. Biochem Biophys Res Commun 411: 523-529, 2011.

13. Sluijter JP, Pulskens WP, Schoneveld AH, Velema E, Strijder CF, Moll F, de Vries JP, Verheijen J, Hanemaaijer R, de Kleijn DP and Pasterkamp G: Matrix metalloproteinase 2 is associated with stable and matrix metalloproteinases 8 and 9 with vulnerable carotid atherosclerotic lesions: A study in human endarterectomy specimen pointing to a role for different extracellular matrix metalloproteinase inducer glycosylation forms. Stroke 37: 235-239, 2006.

14. George J, Harats D, Gilburd B, Afek A, Shaish A, Kopolovic J and Shoenfeld Y: Adoptive transfer of beta(2)-glycoprotein I-reactive lymphocytes enhances early atherosclerosis in LDL receptor-deficient mice. Circulation 102: $1822-1827,2000$

15. George J, Afek A, Gilburd B, Shoenfeld Y and Harats D: Cellular and humoral immune responses to heat shock protein 65 are both involved in promoting fatty-streak formation in LDL-receptor deficient mice. J Am Coll Cardiol 38: 900-905, 2001.

16. Cobbold SP, Castejon R, Adams E, Zelenika D, Graca L, Humm S and Waldmann H: Induction of Foxp $3^{+}$regulatory $\mathrm{T}$ cells in the periphery of $\mathrm{T}$ cell receptor transgenic mice tolerized to transplants. J Immunol 172: 6003-6010, 2004.

17. Yang L, Chu Y, Wang Y, Zhao X, Xu W, Zhang $\mathrm{P}$, Liu X, Dong S, He W and Gao C: siRNA-mediated silencing of Wnt5a regulates inflammatory responses in atherosclerosis through the MAPK/NF-kB pathways. Int J Mol Med 34: 1147-1152, 2014.

18. Huang G, Lv J, Li T, Huai G, Li X, Xiang S, Wang L, Qin Z, Pang J, Zou B and Wang Y: Notoginsenoside R1 ameliorates podocyte injury in rats with diabetic nephropathy by activating the PI3K/Akt signaling pathway. Int J Mol Med 38: 1179-1189, 2016.

19. Brattelid T, Winer LH, Levy FO, Liestøl K, Sejersted OM and Andersson KB: Reference gene alternatives to Gapdh in rodent and human heart failure gene expression studies. BMC Mol Biol 11: 22, 2010.

20. Tsai BY, Suen JL and Chiang BL: Lentiviralmediated Foxp3 RNAi suppresses tumor growth of regulatory $\mathrm{T}$ cell-like leukemia in a murine tumor model. Gene Ther 17: 972-979, 2010.

21. Ziegler SF: FOXP3: Of mice and men. Annu Rev Immunol 24: 209-226, 2006.

22. van Puijvelde GH, Hauer AD, de Vos P, van den Heuvel R, van Herwijnen MJ, van der Zee R, van Eden W, van Berkel TJ and Kuiper J: Induction of oral tolerance to oxidized low-density lipoprotein ameliorates atherosclerosis. Circulation 114: 1968-1976, 2006.

23. Skoog T, Dichtl W, Boquist S, Skoglund-Andersson C, Karpe F, Tang R, Bond MG, de Faire U, Nilsson J, Eriksson P and Hamsten A: Plasma tumour necrosis factor-alpha and early carotid atherosclerosis in healthy middle-aged men. Eur Heart J 23: 376-383, 2002.

24. Bruunsgaard H, Skinh øj P, Pedersen AN, Schroll M and Pedersen BK: Ageing, tumour necrosis factor-alpha (TNF-alpha) and atherosclerosis. Clin Exp Immunol 121: 255-260, 2000.

25. Ridker PM, Rifai N, Stampfer MJ and Hennekens CH: Plasma concentration of interleukin- 6 and the risk of future myocardial infarction among apparently healthy men. Circulation 101: $1767-1772,2000$

26. Ridker PM, Rifai N, Pfeffer M, Sacks F, Lepage S and Braunwald E: Elevation of tumor necrosis factor-alpha and increased risk of recurrent coronary events after myocardial infarction. Circulation 101: 2149-2153, 2000.

27. Huber SA, Sakkinen P, Conze D, Hardin N and Tracy R: Interleukin-6 exacerbates early atherosclerosis in mice. Arterioscler Thromb Vasc Biol 19: 2364-2367, 1999.

28. Kirii H, Niwa T, Yamada Y, Wada H, Saito K, Iwakura Y, Asano M, Moriwaki H and Seishima M: Lack of interleukin-1beta decreases the severity of atherosclerosis in ApoE-deficient mice. Arterioscler Thromb Vasc Biol 23: 656-660, 2003.

29. Libby P, Ridker PM and Maseri A: Inflammation and atherosclerosis. Circulation 105: 1135-1143, 2002. 
30. Zhou X, Nicoletti A, Elhage R and Hansson GK: Transfer of CD4(+) T cells aggravates atherosclerosis in immunodeficient apolipoprotein E knockout mice. Circulation 102: 2919-2922, 2000.

31. Ochs HD, Ziegler SF and Torgerson TR: FOXP3 acts as a rheostat of the immune response. Immunol Rev 203: 156-164, 2005

32. Mor A, Luboshits G, Planer D, Keren G and George J: Altered status of CD4(+)CD25(+) regulatory T cells in patients with acute coronary syndromes. Eur Heart J 27: 2530-2537, 2006.

33. Brunkow ME, Jeffery EW, Hjerrild KA, Paeper B, Clark LB Yasayko SA, Wilkinson JE, Galas D, Ziegler SF and Ramsdell F: Disruption of a new forkhead/winged-helix protein, scurfin, results in the fatal lymphoproliferative disorder of the scurfy mouse. Nat Genet 27: 68-73, 2001.
34. Gu L, Okada Y, Clinton SK, Gerard C, Sukhova GK, Libby P and Rollins BJ: Absence of monocyte chemoattractant protein-1 reduces atherosclerosis in low density lipoprotein receptor-deficient mice. Mol Cell 2: 275-281, 1998.

35. Harrington JR: The role of MCP-1 in atherosclerosis. Stem Cells 18: 65-66, 2000.

36. Pasterkamp G, Schoneveld AH, Hijnen DJ, de Kleijn DP, Teepen $\mathrm{H}$, van der Wal AC and Borst C: Atherosclerotic arterial remodeling and the localization of macrophages and matrix metalloproteases 1,2 and 9 in the human coronary artery. Atherosclerosis 150: 245-253, 2000.

37. Gough PJ, Gomez IG, Wille PT and Raines EW: Macrophage expression of active MMP-9 induces acute plaque disruption in apoE-deficient mice. J Clin Invest 116: 59-69, 2006. 